\title{
Efectos favorables de la actividad física regular motivada en el trabajo sobre la tensión arterial y el perfil lipídico
}

\author{
Beneficial effects of regular physical activity motivated at work on blood pressure \\ and lipid status
}

Alfonso José Hernán', Skogstad Marit', Skare Øivind', Mamen Asgeir², Lunde Lars-Kristian³, Einarsdottirr Elin ${ }^{4}$,
Øvstebø Reidun ${ }^{5}$, Ulvestad Bente 6

1. Departamento de Medicina Ocupacional y Epidemiología. Instituto Nacional de Medicina Ocupacional. Boks 8149 Dep. 0033 Oslo, Norvega.

2. Colegio Universitario de Salud de Noruega. PB 1190 Sentrum, 107 Oslo, Noruega.

3. Departamento de Psicología y Fisiología del Trabajo. Instituto Nacional de Medicina Ocupacional. Boks 8149 Dep. 0033 Oslo, Norvega.

4. Departamento de Química y Biología Ocupacional. Instituto Nacional de Medicina Ocupacional. Boks 8149 Dep. 0033 Oslo, Norvega.

5. Sección de Investigación de Biología Celular. Departamento de Medicina Bioquímica. Hospital Universitario de Oslo.

6. Servicio de Salud Ocupacional, Mesta AS. Postboks 253, 1326 Lysaker. Norvega

Recibido: 07-04-15

Aceptado: 19-05-15

\section{Correspondencia}

José Hernán Alfonso, STAMI

Telefono: +4723195388, fax: +4723195205,

Correo electrónico: jose.alfonso@stami.no

Resumen

Introducción: La inactividad física se ha incrementado de forma considerable en la sociedad actual pudiendo acarrear efectos perjudiciales para la salud. El ejercicio físico regular motivado en el trabajo podría ser una intervención de promoción de la salud y preventiva con beneficios a corto y largo plazo, por lo que es importante documentar sus efectos fisiológicos.

Material y métodos: Estudio de corte transversal que representa la primera parte de un estudio prospectivo donde 121 trabajadores viales, de mantenimiento y de oficina (42 mujeres y 79 hombres) recibieron un programa de motivación de actividad física. Se registró el grado de actividad física previa y actual. Se estudió el índice de masa corporal (IMC), la frecuencia cardíaca (FC), tensión arterial sistólica y diastólica (TAS, TAD), el pérfil lipídico, niveles de proteína C reactiva (PCR) y hemoglobina glicosilada (HbA1c). La captación máxima de oxígeno fue cuantificada por medio de ergoespirometría. Análisis de regresión lineal fueron utilizados para estudiar asociaciones entre las variables de estudio con la respectiva inclusión de variables de confusión.

Resultados: Las mujeres presentaron un pérfil lipídico más favorable y valores de IMC y tensión arterial menores en comparación con los hombres. La actividad física regular más de dos veces por semana estuvo asociada a niveles menores de colesterol total $(\mathrm{p}=0,013$ para $2-3$ veces $/$ semana; $\mathrm{p}=0,025$ para $\geq 4$ vez / semana) y lipoproteínas de baja densidad (LDL) ( $p=0,015$ para 2-3 veces/emana; $p=0,022$ para $\geq 4$ veces / semana). Los valores de TAD eran más bajos en el grupo de los que reportó actividad física regular al menos cuatro veces por semana $(p=0,026)$. El efecto de la actividad física regular sobre la TAD fue más 
evidente en hombres que en mujeres $(\mathrm{p}=0,01)$. Los niveles autoreportados de actividad física mostraron correlación con los niveles de captación máxima de oxígeno medidos.

Conclusión: El pérfil lipídico y los niveles de tensión arterial fueron más favorables en aquellos individuos que reportaron actividad física de forma regular a comparación de aquellos con bajos niveles de actividad física semanal. La actividad física autoreportada se hallaba asociada a los niveles de captación máxima de oxígeno medidos.

Med Segur Trab (Internet) 2015; 61 (239) 162-171

Palabras clave: Actividad física, salud cardiovascular, captación máxima de oxígeno, tensión arterial, promoción de la salud por medio del trabajo, trabajo.

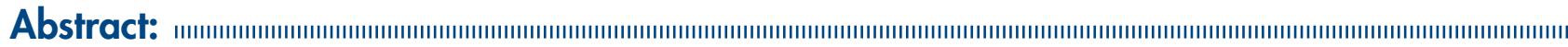

Introduction: Physical inactivity is increasing considerably in the modern society and may lead to adverse health effects. Physical exercise motivated at work could be a health promotion and preventive intervention at work with short and long-term benefits. Therefore it is important to document its physiological effects.

Material and methods: This cross-sectional study represents the first part of a prospective study where 121 road workers, maintenance and office (42 women and 79 men) participated in a motivational program for physical activity. Background data and level of physical activity were collected by a questionnaire. Body mass index (BMI), Maximal oxygen uptake, blood pressure, and blood samples (lipids, C-reactive protein and glycosylated hemoglobin) were obtained. Linear regression analysis was performed to study associations between the study variables with the inclusion of confounding variables.

Results: A more favorable lipid status, BMI values, and lower blood pressure were found among women. Regular physical activity more than twice a week was associated with lower levels of total cholesterol ( $\mathrm{p}=0,013$ twice a week; $\mathrm{p}=0,025$ more than 4 times a week), and low density lipoprotein (LDL) ( $\mathrm{p}=0,015$ twice a week; $\mathrm{p}=0,022$ more than 4 times a week). Diastolic blood pressure (dBP) was lower in the group who reported regular physical activity at least four times a week $(p=0,026)$. The effect of regular physical activity on $\mathrm{dBP}$ was more evident in men than in women $(\mathrm{p}=0,01)$. The intensity of self-reported physical activity was associated with the measured levels of maximal oxygen uptake.

Conclusion: Exercise several times a week was associated with a more favorable lipid status and lower blood pressure compared to lower weekly activity. The intensity of self-reported physical activity was associated to the measured levels of maximal oxygen uptake.

Med Segur Trab (Internet) 2015; 61 (239) 162-171

Key words: Work motivated physical activity, cardiovascular bealth maximal oxygen uptake, blood lipids, blood pressure, bealth promotion at work, work. 


\section{INTRODUCCIÓN}

La Investigación de Trabajadores del Transporte de Londres de $1953^{1}$ fue el primer estudio que sugirió una correlación entre la la inactividad física y el riesgo cardíaco. A partir de 1980 una serie de estudios de seguimiento demostraron que la actividad física, en parte basada en información autorreportada, previene y mejora enfermedades relacionadas con el estilo de vida $^{2,3}$. Se prestó ademas mucha atención al efecto protector de la actividad física contra el desarrollo de la diabetes. Actividades de alta intensidad, tales como natación, tenis y el trote tendrían mayor efecto que actividades de baja intensidad $^{3}$.

Posteriormente, estudios prospectivos poblacionales Norteaméricanos investigaron la salud cardiovascular y mortalidad con seguimientos de hasta 12 años ${ }^{4-6}$. La tasa de mortalidad a causa de enfermedades cardiovasculares fue tres veces mas alta en los hombres con índice de masa corporal (IMC) mayor a 30 en comparación con individuos de peso adecuado; mientras que la mortalidad general fue dos veces mayor en el grupo con IMC $<30$. No obstante, el entrenamiento físico regular en hombres obesos contribuyó a una menor mortalidad debido a enfermedades cardiovasculares y cáncer ${ }^{4}$.

En un estudio reciente ${ }^{7}$ que incluyo un seguimiento por 10 años de 624827 ciudadanos norteamericanos, diez minutos diarios de entrenamiento aumentaron la esperanza de vida por lo menos dos años, mientras que un hora diaria estuvo asociado a un aumento en la esperanza de vida de entre cuatro y cinco años. ${ }^{7}$ Estos hallazgos acuerdan con resultados del "Estudio de Oslo" donde los hombres que entrenaban diariamente tenían $40 \%$ menor mortalidad que los sedentarios ${ }^{8}$.

Sumado a los hallazgos anteriores, la evidencia existente sugiere que la incidencia de infarto de miocardio es mayor en personas con ocupaciones sedentarias ${ }^{9}$. Además, la función cardiorrespiratoria en profesiones tales como administración, ventas e industria alimentaria se hallaría reducida? .

En Noruega cada vez mas son los empleadores y servicios de medicina ocupacional que realizan intervenciones para promocionar la actividad física de los empleados. Sin embargo, los efectos fisiológicos de estos programas de motivación aún no se han evaluado de forma objetiva. Por lo tanto, por medio de este estudio nos propusimos investigar de forma prospectiva los efectos fisiológicos de la actividad física que es motivada en el trabajo. Los resultados transversales de la primer fase del estudio serán presentados y discutidos en el presente artículo.

\section{MATERIAL Y MÉTODOS}

\section{Populación de estudio}

Una muestra de 285 trabajadores viales y de mantenimiento como así también oficinistas participaron en un programa de motivación de la actividad física («Dytt®») llevado a cabo en el lugar de trabajo. Una submuestra de 121 participantes fueron examinados por medio de un control de salud tanto antes como después del programa de motivación.

El programa incluyó el registro diario en una base de datos de la cantidad de pasos al caminar o correr por medio de un contador de pasos. Para otros tipos de actividad física tales como la natación o el ciclismo se calculan hacia "pasos" por medio de una calculadora. Los participantes tenían la posibilidad de establecer equipos de entrenamiento y competir entre ellos, siendo las prestaciones individuales como grupales premiadas al final de la intervención.

Los controles de salud incluyeron mediciones de la tensión arterial y parámetros sanguíneos. En total participaron 79 hombres, de los cuales 27 eran trabajadores viales y 
de mantenimiento calificados y 42 mujeres. La capacidad máxima de oxígeno $\left(\mathrm{VO}_{2} \max \right)$ fue medida en un subgrupo de 53 personas (32 hombres y 21 mujeres: 39 con nivel educativo de colegio secundario/ o universitario completo).

\section{Cuestionario}

Un cuestionario fue administrado durante el control de salud por un médico o enfermero ocupacional. Las variables incluidas fueron: antecedentes personales, estado de salud el día de la examinación médica, nivel educativo, motivación para realizar actividad física, y frecuencia semanal de la misma en el tiempo libre.

\section{Medición de tensión arterial (TA)}

Luego de cinco minutos de reposo se realizaron tres mediciones de TA en el brazo izquierdo, con un intervalo de un minuto entre las mediciones. El promedio de las dos últimas mediciones de tensión arterial sistólica y diastólica fue utilizado en los análisis estadísticos. El instrumento de medición utilizado fue BpTRU ® (Bp TRU medical devices, Coquitlam, Canada).

\section{Los análisis de sangre}

El suero para el análisis de los lípidos [colesterol, lipoproteína de alta densidad (HDL), lipoproteínas de baja densidad (LDL)] y la proteína C-reactiva (CRP) se extrajo de tubos de gel que fueron centrifugados a 35 x1000 rpm durante 15 minutos dentro de los 60 minutos luego de la extracción. Para los análisis de hemoglobina glicosilada (HbA1c) la sangre se conservó en tubos con EDTA. Las muestras fueron enviadas por correo al Departamento de Bioquímica Médica del Hospital Universitario de Oslo, Ullevål y se analizaron dentro de las 24 horas.

\section{Proteína $\mathbf{C}$ reactiva}

La proteína $\mathrm{C}$ reactiva fue cuantificada por medio de tests inmunoturbidimétricos potenciados por partículas por medio de la plataforma analítica modular Cobas 8000 (Roche Diagnostics, www.roche.com). El coeficiente de variación analítica fue del 8,0\%.

\section{Pérfil lipídico}

Los niveles de colesterol, HDL y LDL en suero fueron cuantificados con un método enzimático colorimétrico en la plataforma analítica modular Cobas 8000. Los coeficientes de variación analítica son 3.0, 4,0 y 3,5 \% respectivamente.

\section{Hemoglobina glicosilada (HbA1c)}

La hemoglobina glicosilada fue analizada a partir de la sangre en solución de EDTA con el analizador Tosoh G7 que se basa en el principio de cromatografía líquida de alta eficacia como principio de separación. El coeficiente de variación fue 1,7\%.

\section{La captación máxima de oxígeno $\left(\mathrm{VO}_{2 \max }\right)$}

La captación máxima de oxígeno $\left(\mathrm{VO}_{2}\right.$ máx $)$ fue evaluada a través de una prueba graduada en cicloergómetro (Monark $875 \mathrm{E}$, Vansbro, Suecia). Con una carga inicial de $70 \mathrm{~W}$ por ciclo, los sujetos pedalearon a una frecuencia de 70 ciclos por minuto (RPM). La resistencia se fue aumentando 35 watt por cada minuto hasta el agotamiento (cadencia $<65$ RPM). El consumo de oxígeno se midió continuamente por medio del analizador metábolico Cosmed K4b2 (Cosmed Srl, Roma, Italia) y el $\mathrm{VO}_{2 \text { máx }}$ se determinó a partir del valor máximo durante 30 segundos en la conclusión de la prueba.

\section{Aspectos éticos}

El estudio ha sido aprobado por el Comité Regional de Ética de Oslo (2014/1521). Todos los participantes fueron informados sobre el estudio con antelación y dieron su consentimiento escrito para participar. 


\section{Análisis estadísticos}

El efecto de la actividad física sobre cada una de las variables de estudio (PCR, colesterol total, HDL, LDL, HbA1c, tensión arterial sistólica y diastólica) fue determinado mediante análisis de regresión lineal. Se realizaron ajustes por edad, sexo y educación (secundaria-universitaria conclusa/o inconclusa). No se han realizado ajustes según hábitos tabáquicos debido a que el efecto del tabaquismo no fue significativo $(\mathrm{p}>0,2)$ en todos los análisis. Se realizaron ajustes estadísticos con respecto al IMC para determinar en que medida el efecto del ejercicio podría ser mediado por el IMC. Se analizaron tambien las interacciones entre actividad física y las demás covariables (sexo, edad y educación). La inclusión de los términos de interacción se basó en pruebas de coeficiente de probabilidades y regresión por pasos (combinación de selección de variables hacia atrás y adelante con el punto de inicio en un modelo completo con todo el término de interacción incluido). Todos los ensayos estadísticos se realizaron con el programa $\mathrm{R}$ (http://www.r-project.org).

\section{RESULTADOS}

Las características generales de los participantes se presentan en la Tabla 1.

Tabla 1. Características generales y variables de resultado de la muestra de estudio, según sexo

\begin{tabular}{|c|c|c|}
\hline & \\
\hline & $\begin{array}{c}\text { Hombres }(\mathrm{N}=79)^{\mathrm{a}} \\
\text { Promedio (SD) }\end{array}$ & $\begin{array}{c}\text { Mujeres (N=42) } \\
\text { Promedio (SD) }\end{array}$ \\
\hline Edad (años) & $41,9(11,9)$ & $42,5(12,6)$ \\
\hline Índice de masa corporal (IMC) $\left(\mathrm{kg} / \mathrm{m}^{2}\right)$ & $26,3(4,0)$ & $24,4(3,1)^{*}$ \\
\hline Fumadores $^{\mathrm{a}}$ & $12(15 \%)$ & $0^{*}$ \\
\hline Educación secundaria/universitaria ${ }^{a}$ & $42(53 \%)$ & $36(86 \%) *$ \\
\hline Tensión arterial sistólica $(\mathrm{mmHg})$ & $120(13)$ & $112(16)^{*}$ \\
\hline Tensión arterial diastólica (mmHg) & $77(9)$ & $73(8)^{*}$ \\
\hline PCR $(\mathrm{mg} / \mathrm{L})^{\mathrm{c}}$ & $1,9(2)$ & $1,9(1,9)$ \\
\hline Colesterol $(\mathrm{mmol} / \mathrm{L})^{\mathrm{c}}$ & $5,1(1,1)$ & $5,4(1,1)$ \\
\hline $\mathrm{HDL}(\mathrm{mmol} / \mathrm{L})^{\mathrm{c}}$ & $1,4(0,3)$ & $1,9(0,4)^{*}$ \\
\hline $\mathrm{LDL}(\mathrm{mmol} / \mathrm{L})^{\mathrm{c}}$ & $3,2(0,9)$ & $3,2(0,9)$ \\
\hline $\operatorname{HbA1c}(\mathrm{mmol} / \mathrm{L})^{\mathrm{c}}$ & $5,3(0,4)$ & $5,2(0,3)$ \\
\hline $\begin{array}{l}\text { "Diferencias significativas entre los sexos, } \mathrm{p}<0,05 \\
{ }^{\mathrm{a}} \text { Cantidad total (porcentaje) } \\
{ }^{\mathrm{b}} \mathrm{N}=41 \text { para IMC, PCR, LDL, HDL, COL } \\
{ }^{\mathrm{c}} \mathrm{N}=78 \text { para PCR, } \mathrm{n}=77 \text { for LDL, HDL y colesterol, } \mathrm{n}=75 \text { para HbA1c }\end{array}$ & & \\
\hline
\end{tabular}

De acuerdo a los autorreportes de actividad física regular, 45 participantes reportaron un hábito de entrenamiento con una frecuencia de una vez por semana, 57 individuos entre dos y tres veces por semana mientras que 19 entrenaban al menos cuatro veces por semana. La figura 1 muestra la relación entre la actividad física reportada y la captación máxima de oxígeno $\left(\mathrm{VO}_{2 \max }\right)$ (en relación al sexo y edad) que fue medida en 53 sujetos. 
Figura 1. Capacidad máxima de oxígeno en relación a la actividad física autoreportada

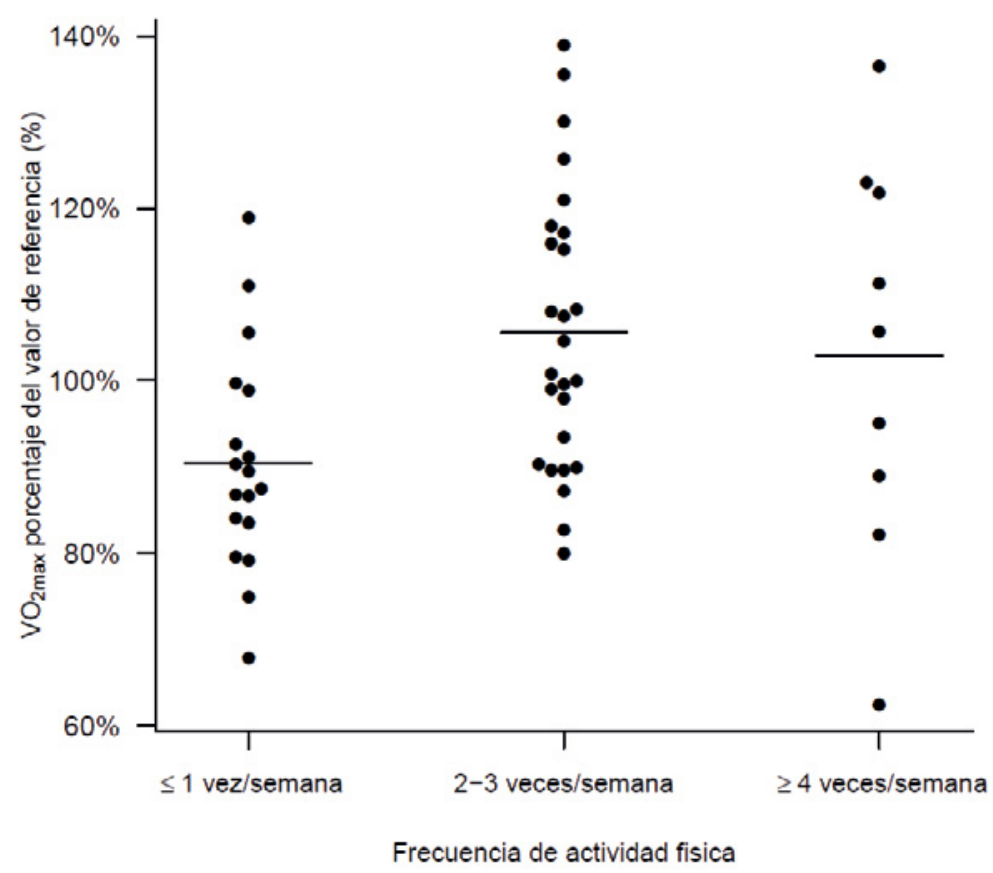

La mayoría de los participantes en el grupo que entenaba menos de dos veces por semana eran trabajadores viales y mantenimiento del sexo masculino. En términos generales, el $64 \%$ de la población estudiada tenía la escuela secundaria o educación universitaria completa. Un 55\% de los 45 que entrenaban menos de dos veces por semana tenían el mismo nivel educativo anteriormente mencionado. A pesar de que los trabajadores calificados $(\mathrm{N}=27)$ tenían la misma edad que los trabajadores de oficina $(\mathrm{N}=52)$, los niveles de HDL eran inferiores entre el primer grupo (IC del 95\%: 0,05, 0,36; $\mathrm{p}=0,013$ ) mientras que los niveles de PCR se hallaban elevados ( $\log$ transformado, $\mathrm{p}=0,022$ ).

Las mujeres presentaron IMC, niveles de tensión arterial inferiores, y un perfil lipídico más favorable (valores mas elevados de HDL) que los hombres (Tabla 1). Esta diferencia se mantuvo significativa aún después de ajustes estadísticos en relación a la edad, educación e intensidad de actividad física. Los niveles de ejercicio físico eran menores entre los trabajadores calificados que entre los de oficina ( $48 \%$ entrenaba menos de dos días por semana).

Los niveles totales de colesterol se hallaban reducidos de forma significativa en relación a la frecuencia creciente de actividad física (IC del 95\% $-0.95,-0.12 ; \mathrm{p}=0,013$ para 2-3 veces / semana y el 95\% CI $-1,23,-0,09 ; \mathrm{p}=0,025$ para $\geq 4$ veces / semana) y LDL (IC $195 \%-0.80,-0.09 ; \mathrm{p}=0,015$ para $2-3$ veces / semana y el IC 95\% -1,7, -0,9; $\mathrm{p}=0,022$ para $\geq 4$ veces / semana) en análisis ajustados por edad, sexo y educación (Figura 2). 
Figura 2. Intensidad de actividad física autoreportada y niveles séricos de colesterol

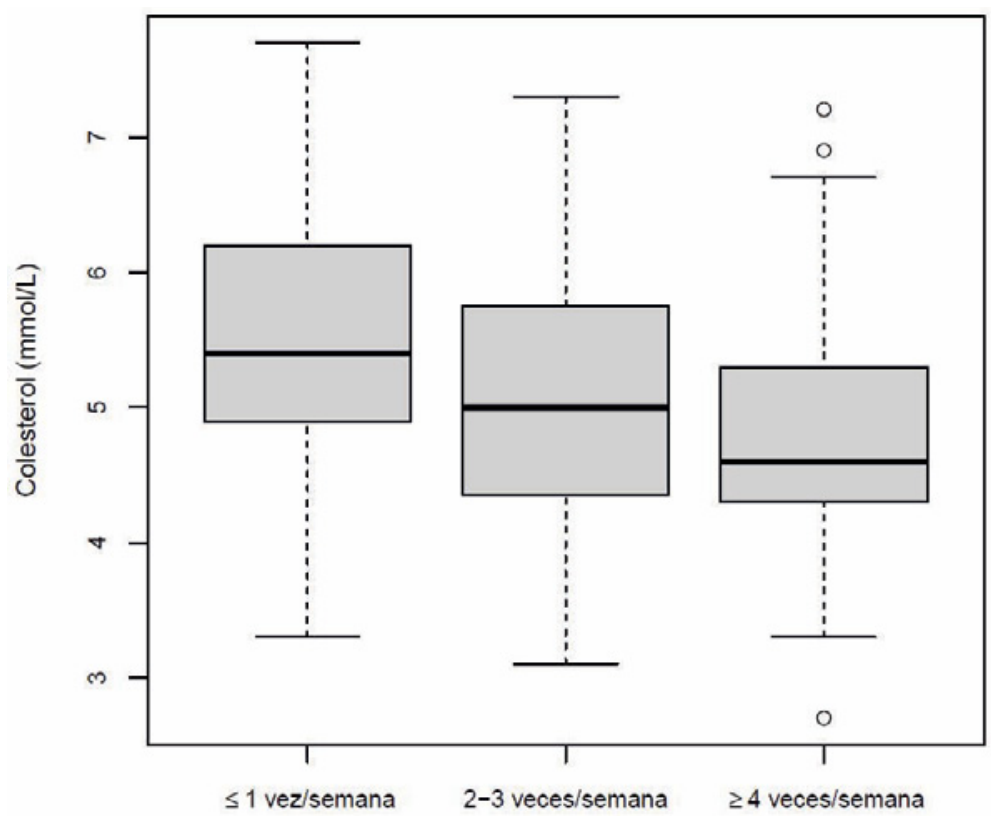

No se observó ninguna asociación entre los niveles de PCR y la frecuencia de actividad física reportada. Los niveles de PCR se hallaban fuertemente asociados a los valores de IMC, pudiendo sugerir que un incremento en los IMC conlleva un aumento de la PCR (Figura 3).

Figura 3. IMC y tendencia inflamatoria medida por medio de PCR

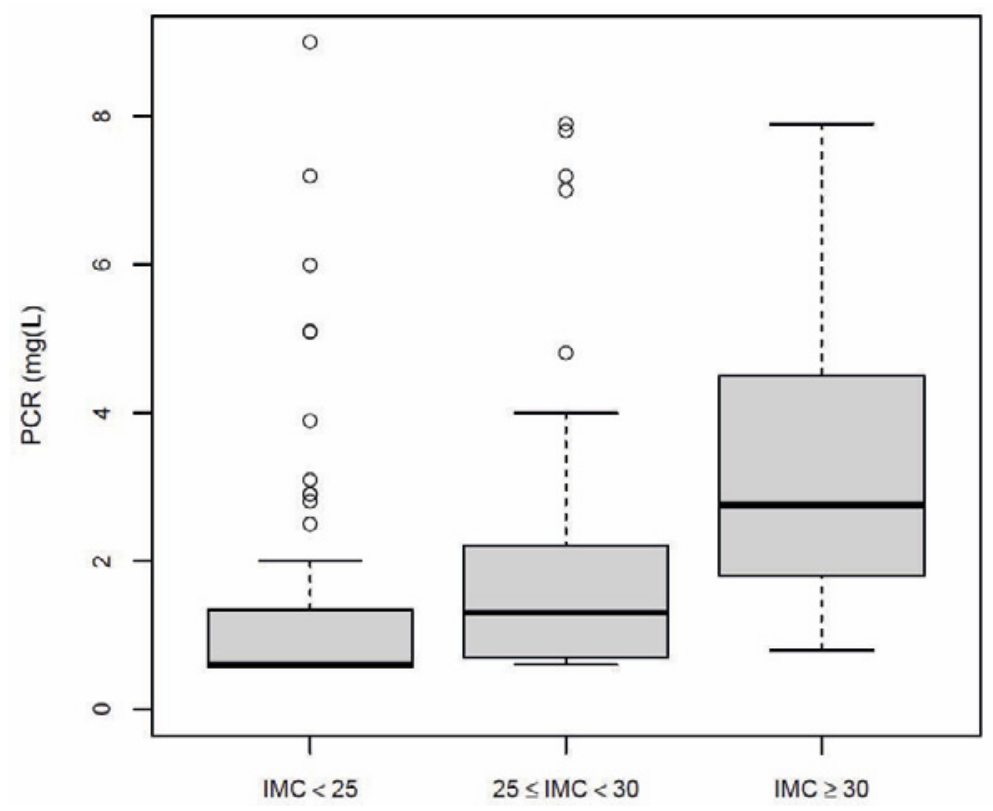

Los niveles de HbA1c no se vieron afectados de forma significativa por el ejercicio regular. Sin embargo se pudo observar que a mayor edad, los valores de HbA1c eran más altos (resultados no mostrados).

La actividad física con una frecuencia de ( $\geq 4$ veces / semana) afectó a la tensión arterial diastólica de forma favorable (IC del 95\% -8,86, -0,61; p = 0,026). El aumento del IMC de una unidad resultó en un aumento de la tensión arterial sistólica (95\% CI 0,09,1,29; $\mathrm{p}=0,027)$ y diastólica $(95 \%$ ki- 0.14, -0.90; $\mathrm{p}=0,009)$. Además un aumento del IMC 
estuvo asociado a niveles más altos de la PCR (95\% KI - 0,04,-0,24; p = 0,006) como así también a una reducción de HDL $(95 \%$ KI -0,05, -0,01; p = 0,002).

Estos resultados sugerirían que algunos de los efectos de la actividad física regular estarían mediados por una reducción del IMC. Los niveles promedio del IMC para los participantes pertenecientes a las diferentes categorías de actividad física fueron los siguientes 26,3 ( $\leq 1 \mathrm{vez} /$ semana), 25,4 (2-3 veces/semana) y 24,7 ( $\geq 4$ veces/semana). Estas diferencias en el IMC no fueron significativas tanto en el modelo sin ajustes, como en aquel ajustado por edad, sexo y educación.

Los efectos de la actividad física regular sobre el colesterol y LDL no parecerían ser mediados por el IMC, pero el efecto de la actividad física frecuente sobre la tensión arterial diastólica se redujo al realizar ajustes de acuerdo al IMC, de -4,74 til -3,94 (IC del $95 \%-8,01,-0,13 ; \mathrm{p}=0,06)$.

Se ha examinado también si los efectos de interacción afectan las variables de resultado. En comparación con las mujeres, el efecto de la actividad física semanal $(\geq 4$ veces/semana) sobre la tensión arterial diastólica fue mayor entre los hombres (95\% -20,8 CI, $-3,0 ; p=0,01)$.

\section{DISCUSIÓN}

En este estudio transversal de 121 funcionarios y trabajadores de mantenimiento vial, la actividad física autorreportada con una frecuencia de dos o más veces por semana influenció de forma favorable el perfil lipídico y los niveles de tensión arterial. Las mujeres obtuvieron valores más favorables de HDL y tensión arterial que los hombres, incluso luego de realizar ajustes por educación, edad y nivel de condición física.

Los niveles de LDL y colesterol total eran inferiores entre los que hacían ejercicio al menos dos veces por semana en comparación con los que reportaron menor frecuencia. Hallazgos similares fueron descriptos en ensayos clínicos previos. Un meta-análisis de estudios científicos sobre personas con dislipidemia ha demostrado que una intervención con actividad física por varias semanas estaba asociada a un incremento del HDL y reducción del colesterol total/LDL ${ }^{10}$.

En base a los resultados obtenidos, el efecto de la actividad física semanal frecuente sobre la tensión arterial diastólica fue mayor para los hombres que para las mujeres. Para el grupo en general, la tensión arterial fue mas baja entre aquellos individuos que reportaron actividad física regular y frecuente. Similares hallazgos fueron documentados en estudios de personas con hipertensión arterial y síndrome metabólico ${ }^{11}$. Otros estudios han documentado que el ejercicio físico tendría un un efecto beneficioso sobre la tensión arterial de normotensos ${ }^{11}$. La reducción de la tensión arterial en la población general podría tener un efecto sobre las mortalidad general a causa de enfermedades cardiovasculares. ${ }^{12}$ Las directrices europeas para la enfermedades cardiovasculares afirman que la prevención primaria sería mas beneficiosa que el tratamiento de las mismas $^{13}$.

La obesidad y los bajos niveles de aptitud física estarían relacionados con cuadros pro-inflamatorios sistémicos ${ }^{14}$. En este estudio transversal, se encontró una clara correlación entre el aumento del IMC y la inflamación medida por las concentraciones séricas de PCR. Sin embargo no se halló una clara asociación entre los grados de actividad física y la reducción de este marcador inflamatorio. Un estudio de seguimiento que se halla bajo ejecución podría elucidar asociaciones plausibles entre la actividad física regular con la actividad inflamatoria.

Un tercio de los hombres en el presente estudio correspondían a trabajadores viales especialmente calificados para el mantenimiento de carreteras, con algunas tareas manuales; pero con mayor grado de actividad sedentaria debido al manejo de grandes camiones, grúas y otras máquinas. Este grupo tenía niveles mayores de PCR, bajos de HDL 
y menor actividad física que el resto. Estos sujetos podrían además hallarse expuestos a micropartículas que podrían afectar la salud cardiovascular de forma negativa ${ }^{15}$. Esto es motivo de preocupación, ya que estudios de seguimiento de trabajadores con ocupaciones manuales han sugerido un aumento de la mortalidad entre aquellos físicamente inactivos, y menor mortalidad entre los que practican actividad física regularmente ${ }^{16,17}$.

El diseño de esta investigación, no consideró otros factores de riesgo correspondientes al estilo de vida tales como nutrición, relaciones familiares, y factores genéticos. Tanto la selección de individuos sanos y motivados para entrenar como así tambien una clasificación errónea de la actividad física autorreportada no se pueden descartar en el presente estudio. Estudios futuros que incluyen el seguimiento longititudinal por un periodo de ocho semanas y doce meses nos permitirán comprender más acerca de los efectos fisiológicos de la actividad física motivada en el trabajo.

A pesar de las limitaciones metodológicas, consideramos que los hallazgos de este estudio transversal ofrecen indicios de que la actividad física regular motivada en el trabajo proporcionaría beneficios fisiológicos para la salud cardiovascular. Por lo tanto el lugar de trabajo podría ser un ámbito ideal para la promoción de la salud cardiovascular.

\section{CONCLUSIÓN}

La actividad física regular motivada en el trabajo contribuiría a un perfil lipídico favorable y a la reducción de la tensión arterial diastólica. La actividad física regular debería ser aconsejada a aquellos trabajadores con profesiones manuales y sedentarias.

\section{REFERENCIAS BIBLIOGRÁFICAS}

1. Morris JN, Heady JA, Raffle PA, Roberts CG, Parks JN. Coronary heart-disease and physical activity of work. Lancet. 1953; (265): 1111-1120.

2. Paffenbarger RS Jr., Hyde RT, Wing AL, Hsieh CC. Physical activity, all-cause mortality, and longevity of college alumni. N Engl J Med. 1986; (314): 605-613.

3. Manson JE, Rimm EB, Stampfer MJ, Colditz GA, Willett WC, Krolewski AS, et al. Physical activity and incidence of non-insulin-dependent diabetes mellitus in women. Lancet. 1991; (338): 774-778.

4. Wei M, Kampert JB, Barlow CE, Nichaman M Z, Gibbons LW, Paffenbarger RS Jr, et al. Relationship between low cardiorespiratory fitness and mortality in normal-weight, overweight, and obese men. JAMA. 1999; (282): 1547-1553.

5. Sui X, LaMonte MJ, Laditka JN, Hardin JW, Chase N, Hooker SP, et al. Cardiorespiratory fitness and adiposity as mortality predictors in older adults. JAMA. 2007; (298): 2507-2516.

6. Blair SN, Kampert JB, Kohl HW $3^{\text {rd }}$, Barlow C E, Macera CA, Paffenbarger RS Jr, Gibbons LW. Influences of cardiorespiratory fitness and other precursors on cardiovascular disease and all- cause mortality in men and women. JAMA. 1996; (276): 205-210.

7. Moore SC, Patel AV, Matthews CE, Berrington de Gonzalez A, Park Y, Katki HA, et al. Leisure time physical activity of moderate to vigorous intensity and mortality: a large pooled cohort analysis. PLoS Med. 2012; (9); e1001335.

8. Holme I, Anderssen SA.[Physical activity, smoking and mortality among men who participated in the Oslo studies of 1972 and 2000]In Nowegian. Tidsskr Nor Laegeforen. 2014; (134): 1743-1748.

9. Lewis JE, Clark JD $3^{\text {rd }}$, LeBlanc WG, Fleming LE, Caban-Martinez AJ, Arheart KL, et al. Cardiovascular fitness levels among American workers. J Occup Environ Med. 2011; (53): 1115-1121.

10. Leon AS, Sanchez OA. Response of blood lipids to exercise training alone or combined with dietary intervention. Med Sci Sports Exerc. 2001; (33): S502-15; discussion S528-9.

11. Whelton SP, Chin A, Xin X, He J. Effect of aerobic exercise on blood pressure: a meta-analysis of randomized, controlled trials. Ann Intern Med. 2002; (136): 493-503.

12. Lewington S, Clarke R, Qizilbash N, Peto R, Collins R. Age-specific relevance of usual blood pressure to vascular mortality: a meta-analysis of individual data for one million adults in 61 prospective studies. Lancet. 2002; (360): 1903-1913. 
13. Perk J, DeBacker G, Gohlke H, Graham I, Reiner Z, Verschuren M, et al. European Guidelines on cardiovascular disease prevention in clinical practice (version 2012). The Fifth Joint Task Force of the European Society of Cardiology and Other Societies on Cardiovascular Disease Prevention in Clinical Practice (constituted by representatives of nine societies and by invited experts). Eur Heart J. 2012; (33): 1635-1701.

14. Mohamed-Ali V, Goodrick S, Rawesh A, Katz DR, Miles JM, Yudkin JS, et al. Subcutaneous adipose tissue releases interleukin-6, but not tumor necrosis factor-alpha, in vivo. J Clin Endocrinol Metab. 1997; (82): 4196-4200.

15. Miller MR, Shaw CA, Langrish LP. From particles to patients: oxidative stress and the cardiovascular effects of air pollution. Future Cardiol. 2012; (8): 577-602.

16. Clays E, Lidegaard M, DeBacquer D, Van Herck K, De Backer G, Kittel F, et al. The Combined Relationship of Occupational and Leisure-Time Physical Activity With All-Cause Mortality Among Men, Accounting for Physical Fitness. Am J Epidemiol. 2014;(179):559-566.

17. Holtermann A, Marott JL, Gyntelberg F, Sogaard K, Suadicani P, Mortensen OS, et al. Occupational and leisure time physical activity: risk of all-cause mortality and myocardial infarction in the Copenhagen City Heart Study. A prospective cohort study. BMJ Open. 2012; (2): e000556.

|| 